\title{
Management of brain metastases in elderly patients with lung cancer
}

\author{
Joanna Socha ${ }^{1,2}$, Anna Rychter ${ }^{1}$, Lucyna Kepka ${ }^{1}$ \\ ${ }^{1}$ Department of Radiotherapy, Military Institute of Medicine, Warsaw, Poland; ${ }^{2}$ Department of Radiotherapy, Regional Oncology Centre, \\ Czestochowa, Poland \\ Contributions: (I) Conception and design: J Socha; (II) Administrative support: None; (III) Provision of study materials or patients: None; (IV) \\ Collection and assembly of data: None; (V) Data analysis and interpretation: None; (VI) Manuscript writing: All authors; (VII) Final approval of \\ manuscript: All authors. \\ Correspondence to: Joanna Socha. Department of Radiotherapy, Military Institute of Medicine, ul. Szaserów 128, 04-141 Warsaw, Poland. \\ Email: sochajoanna@wp.pl.
}

\begin{abstract}
The incidence of brain metastases (BM) is continuing to grow in the elderly population with lung cancer, but these patients are seriously under-represented in clinical trials. Thus, their treatment is not based on the evidence from randomized prospective studies. Age is a well recognized poor prognostic factor for survival in patients with BM from lung cancer, which is reflected in prognostic scales, but its impact on the patients' prognosis reflected by its value in gradually updated grading indices seems to decrease. The reason for poorer outcomes in the elderly is unknown-it may result from the influence of the age per se, simplified staging work-up and suboptimal treatment in this patient subgroup or the excess toxicity of the aggressive anticancer treatment secondary to the impaired physiological regulation mechanisms and comorbidities. The main goal of treatment of BM is to ameliorate neurological symptoms and delay neurological progression, with the focus on the improvement and maintenance of the patients' quality of life. The possible treatment options for BM from lung cancer are whole-brain radiotherapy, stereotactic radiosurgery, surgery, chemotherapy, targeted therapies and best supportive care. The aim of this review is to summarize the problems related to the management of BM in elderly patients with lung cancer, to analyze the value of the above mentioned treatment options, and to provide an insight into the influence of age-related clinical factors on the patients' outcomes.
\end{abstract}

Keywords: Brain metastases (BM); lung cancer; elderly

Submitted May 13, 2020. Accepted for publication Sep 17, 2020.

doi: 10.21037/jtd-2019-rbmlc-05

View this article at: http://dx.doi.org/10.21037/jtd-2019-rbmlc-05

\section{Introduction}

Lung cancer remains the most common cause of brain metastases (BM) (1). The incidence of BM is continuing to grow in the elderly population (2,3), along with increasing life expectancy and prolonged survival even in elderly patients with systemic lung cancer (4). The definition of the elderly, however, seems to change gradually, with a cutoff age moving from 65 (5) to more than 70-75 years of age $(6,7)$. A growing proportion of "very elderly" patients aged 80 years and older, receives active anti-cancer treatment nowadays $(7,8)$, although this age population is absent in clinical trials, and even younger elderly patients (6575 years) are seriously under-represented. On the other hand, most elderly patients have lower performance status, multiple comorbidities, restricted physiological reserves and age-related neurocognitive deficits, which influence therapeutic choices and patients' preferences.

Age is a well recognized poor prognostic factor for survival in patients with lung cancer and $\mathrm{BM}(5,6,9)$. Little is known, however, what is the reason for poorer 
outcomes in the elderly-is it the influence of the age per $s e$, simplified staging work-up and suboptimal treatment in this patient subgroup, or maybe the excess toxicity of the aggressive anticancer treatment secondary to the impaired physiological regulation mechanisms and comorbidities which results in worse outcomes in this specific subgroup?

The aim of this review is to summarize the problems related to the management of BM in elderly patients with lung cancer, and to provide an insight into the influence of age-related clinical factors on the patients' outcomes.

\section{General aspects of BM management in older patients}

The main goal of treatment of BM, regardless of the patients' age is to ameliorate neurological symptoms and delay neurological progression, with the focus on the improvement and maintenance of the patients' quality of life (QoL). Elderly patients, with shorter life expectancy and expected impaired treatment tolerance, are usually treated less aggressively than their younger counterparts $(10,11)$, also the adherence to staging and surveillance guidelines is worse for this subgroup $(11,12)$. However, there is inconclusive data regarding the impact of the aforementioned treatment-related differences on clinical outcomes and toxicity in the case of the elderly as compared to the younger group. A retrospective analysis of all patients who received first line chemotherapy (CHT) for non-small cell lung cancer (NSCLC) in two tertiary cancer centers in Greece, was performed to assess if elderly patients $>70$ years with advanced NSCLC $(\mathrm{N}=107)$ were treated differently compared to their younger counterparts $(\mathrm{N}=138)$ in routine clinical practice, and whether this discordance translated into differences in toxicity and efficacy outcomes (11). Although elderly patients tend to receive suboptimal treatment, with type, intensity and duration of CHT differing significantly between elderly and non-elderly groups, this difference was not reflected in toxicity or clinical outcomes. Contrarily, in the study on 42,323 elderly patients (aged $\geq 65$ years) with NSCLC or small-cell lung cancer (SCLC) from the Surveillance, Epidemiology, and End Results (SEER) database, from years 2002-2007, survival outcomes were significantly better among patients receiving guideline-concordant lung cancer care, and the adjusted lung cancer mortality risk was significantly higher among patients receiving guideline-discordant care [hazard ratio (HR) 1.91, 95\% CI: 1.82-2.00; $\mathrm{P}<0.001]$. Stratified analysis showed significant survival benefit with receipt of guideline-concordant care among patients with NSCLC and patients with SCLC (10). However, there is no data on the differences in diagnostic and therapeutic approach between the elderly and younger patients with BM from lung cancer.

\section{Prognostic scales}

As the outcomes of patients with BM are affected by many factors, a prognostic grading index might resolve some uncertainties and guide personalized treatment decisions. Several grading indices have been developed, namely: (I) the RTOG recursive partitioning analysis (RPA) (5) including patient age, Karnofsky performance status (KPS), control of primary tumor and extracranial metastases, (II) the Score Index for Radiosurgery (SIR) (13) including age, KPS, status of systemic disease, number of lesions, and largest lesion volume, (III) the Basic Score for Brain Metastases (BSBM) (14) including KPS, control of primary tumor, and extracranial metastases, (IV) the graded prognostic assessment (GPA) (9) including patient age, KPS, number of $\mathrm{BM}$ and extracranial metastases, (V) the diagnosis-specific GPA (DS-GPA) (15), i.e., GPA validated and refined with diagnosis-specific prognostic indices and, more recently, 6) the GPA for lung cancer using molecular markers (Lungmol GPA) (6). For SCLC, Bernhardt et al. (16) proposed a new prognostic Brain Metastases from SCLC Score (BMSscore), which included KPS, extracranial disease status (stable $v s$. controlled) and synchronous $v s$. metachronous BM presentation. Recently, Yamamoto et al. (17), proposed the elderly-specific BSBM (ES-BSBM), a modification of BSBM incorporating BM-related factors, i.e., the number of BM (solitary vs. multiple) and cumulative tumor volume $(\leq 15 v s .>15 \mathrm{ccm}$ ), which was shown to be applicable to patients with all primary tumors as well as those 80 years or older. Although all the indices, apart from the (ES-)BSBM, incorporate age as a scoring factor in lung cancer patients, its impact on the patient prognosis reflected by its value in gradually updated grading indices is decreasing. Age older than 65 years excluded patients from Class I RTOG RPA, in the GPA an age $>60$ years resulted in loss of one of four points and two of ten points in SIR, reflecting definitely worse prognosis in the elderly. However, in the most recent Lung-mol GPA index, age $>70$ years results in loss of only 0.5 of four points. NSCLC patients with the same status of driver mutations, KPS, extracranial metastases, and number of $\mathrm{BM}$ are now considered to have the same prognosis irrespective of their age. Median survival ranges from 3.0 
to 46.8 months nowadays (6), as compared with 2.3 to 7.1 months twenty years ago (5). This suggests that in the era of novel less toxic and more effective local and systemic therapies like stereotactic radiosurgery (SRS) vs. wholebrain radiotherapy (WBRT) and targeted therapies (TT) vs. chemotherapy (CHT), the impact of age per se on survival becomes less important. For SCLC, however, the value of the RTOG RPA classes with age and KPS as the main prognostic factors seems still unquestionable. Although the SCLC patients constituted only $4 \%$ in the original RTOG database (5), retrospective studies have confirmed the validity of the prognostic stratification of BM from SCLC according to the RTOG RPA criteria. On the other hand, the largest retrospective study on patients with BM from SCLC that included 229 patients treated with WBRT showed that the above mentioned BMS-score based on three favorable prognostic factors: KPS $>70 \%$, extracranial disease control and synchronous (vs. metachronous) BM presentation was more prognostic than the RPA and DSGPA scores (16). Of note, this new proposed SCLC-specific prognostic index did not include patients' age. However, in the era of rapid expansion of local treatment of BM, the other proposed factors of potential prognostic value, like the initial response to CHT, the number of BM and the timing of their occurrence (synchronous $v s$. metachronous) require further evaluation.

\section{Treatment options}

The possible treatment options for BM from lung cancer are WBRT, SRS, surgery, CHT, TT and best supportive care (BSC)-i.e., no active anticancer treatment.

\section{Radiation therapy: WBRT, SRS}

WBRT that allows not only for local but also for distant intracranial control is a historical standard of treatment for BM. However, in the last decade, none of the three randomized studies comparing the use of SRS alone $v s$. SRS plus WBRT shows any survival benefit in the WBRT arm (18-20). Analysis by age did not reveal any significant survival difference between younger and older patients in these trials. On the other hand, a randomized trial comparing the use of WBRT $v s$. WBRT plus SRS boost showed an increased local control in all patients with the use of SRS boost, and a survival benefit in patients with single metastatic lesion (6.4 vs. 4.9 months, $\mathrm{P}=0.0393$ ) (21). However, secondary analysis of this trial post-stratified by the GPA, showed that irrespective of the number of brain metastases, SRS boost after WBRT resulted in increased survival only in patients with GPA score of 3.5-4.0 (good prognosis); the median survival was 21 months in WBRT + SRS vs. 10.3 months in WBRT (22). The GPA index for lung cancer patients older than 60 years cannot exceed a score of 3.0, for patients with such a score no survival benefit was shown with SRS boost (5.0 months in WBRT+SRS vs. 5.4 months in WBRT alone). Nevertheless, WBRT is gradually being replaced by SRS in current clinical practice, mainly due to the concerns of a detrimental effect of WBRT on neurocognitive functioning $(19,23-26)$ and QoL $(27,28)$. Aged patients are more susceptible to radiation-induced neurotoxicity (29), also the duration of treatment-longer in WBRT - is more relevant in their case. SRS seems to be a reasonable choice due to excellent local control, favorable neurocognitive outcomes, less risk of late side effects, and the absence of adverse effects on patient performance status, also in elderly and carefully selected very elderly patients with BM $(4,7,30,31)$. In retrospective studies good performance status (KPS $\geq 70-80$ ) and stable extracranial disease were associated with survival benefits in elderly patients treated with SRS (8,30-32). Chen et al. (8) reported that in 119 elderly (age 70-79) and very elderly (age $\geq 80$ ) patients with $\mathrm{BM}$, increased acute toxicity was associated with the use of WBRT compared with SRS: fatigue was reported in $87 \%$ vs. $35 \%$ and headaches in $52 \%$ vs. $19 \%$ of patients, respectively. Moreover, $35 \%$ of patients with WBRT $v s$. only $2 \%$ of these with SRS had a documented decline in KPS at the end of treatment, $\mathrm{P}=0.0005$. However, in this retrospective study, selection bias must have impacted toxicity outcomes, because baseline disease extent could influence not only survival outcomes but also patients' and physicians' treatment choices and perception of treatment related toxicity. In a subset study of elderly patients using the JLGK0901Study (33) database, Higuchi et al. (34) compared the SRS treatment results between 693 elderly ( $\geq 65$ years) and 501 non-elderly patients, 912 (76.4\%) of them with lung cancer. They revealed that although median overall survival (OS) time was significantly shorter in the elderly than in the non-elderly patient group (10.3 vs. 14.3 months, respectively, $\mathrm{P}<0.0001$ ) - probably mainly due to significant imbalances in primary cancer sites and KPS between the two groups, all the secondary endpoint results for the elderly group were not inferior to those of the non-elderly group. Actually, both crude and cumulative incidences of tumor recurrence were lower in the elderly than in the non-elderly patient group, which prompted the authors to speculate that the population of 
cancer stem cells probably differed between the two age groups. Also, unexpectedly, both crude and cumulative incidences of SRS-related complications were lower in the elderly than in the non-elderly patient group, although dose de-escalation was not considered for elderly patients and there was no difference in peripheral doses between the two groups. They concluded that elderly BM patients are not unfavorable candidates for SRS alone (34). However, taking into account the ethnic and epidemiological context, extrapolating of the Japanese data to the Western elderly population may not be valid.

Brain necrosis represents the most important late toxicity reported after SRS, leading to neurological complications in $2-32 \%$ of patients, with radiation dose, tumor volume and location of the lesion being the most important predictive variables (35). Although older age ( $\geq 65$ years) is one of the suggested risk factors for radiation necrosis (36), its significant influence on the risk of radionecrosis after SRS was not confirmed in several retrospective cohort studies (37-39) and prospective observational studies $(34,35)$.

\section{Surgery}

The value of surgical resection in combination with radiotherapy (RT) in the treatment of BM patients is well established (40-42), with the benefit of relieving mass effect in large symptomatic lesions, however the risks of brain surgery in elderly patients with BM are not well defined (43). Although the contribution of surgical procedures to morbidity and mortality in the elderly is poorly understood, it seems that neurosurgical removal of BM among selected elderly patients is feasible. However, age older than 80 years and the higher burden of comorbidities are considered to be the most important prognostic factors for postoperative complications (43).

\section{Chemotherapy}

Although the blood-brain barrier (BBB) limits the distribution of some chemotherapeutic agents into the central nervous system (CNS), prospective studies have shown that CHT (platinum doublet or triplet) has the brain response rates ranging from $23 \%$ to $50 \%$ with median survival times of 4 to 12.7 months in the first-line setting without WBRT for BM form NSCLC (44), suggesting that $\mathrm{BBB}$ is likely disrupted by tumor invasion. According to the ESMO guidelines, in NSCLC patients with asymptomatic $\mathrm{BM}$ who have not received prior systemic therapy (CHT or TT), treatment with CHT and deferred WBRT should be considered (45). Administration of CHT in the elderly
NSCLC patients depends mainly on their performance status. Platinum-based CHT is preferred in the elderly with good performance status (WHO 0-1 and selected WHO 2) and adequate organ function, while a single-agent approach might be the recommended treatment for unfit or comorbid patients, who are more likely to present with significantly more treatment-related adverse events (45).

For SCLC patients with known chemosensitivity, CHTresponse rates are even higher than in NSCLC: up to $66-73 \%(46,47)$. In SCLC patients with asymptomatic BM diagnosed at the initial staging, treatment usually starts with $\mathrm{CHT}$, also with the intention of stopping life-threatening extracranial systemic and local disease progression (48). Taking into account the neurocognitive toxicity of WBRT which is most pronounced in the older adults, CHT alone may be a reasonable strategy for the SCLC patients with asymptomatic BM, especially considering that no survival benefit of adding WBRT to CHT was found in 1,615 patients $>75$ years with BM from SCLC (median OS was 5.6 months with WBRT and 6.4 months without, $\mathrm{P}=0.43$ ) (49). However, in the only one randomized trial conducted so far, which compared CHT alone vs. CHT+WBRT 30 Gy in 120 patients with progression in the brain after or during first-line CHT, radiotherapy significantly prolonged the time to disease progression inside the brain $(\mathrm{P}=0.005)$. OS did not differ between the two arms. WHO performance status, and not age, was the only significant prognostic factor for survival (50).

\section{Targeted therapy and Immunotherapy}

Targeted agents (molecular inhibitors) or immunotherapy with monoclonal antibodies have demonstrated far greater intracranial efficacy than conventional CHT, changing the paradigms in the treatment of NSCLC patients with BM. Patients with driver mutations (e.g., EGFR, ALK) have a markedly improved survival $v s$. those without. In these patients with clinically asymptomatic BM, new-generation TT may restore control of brain disease and delay cranial radiotherapy (51), which is of importance in the elderly, more susceptible to radiation-induced neurotoxicity.

The benefit in progression-free survival conferred by EGFR tyrosine kinase inhibitors (TKIs) was consistent across all the phase III randomized control trials comparing EGFR-TKIs to CHT (52-56) and was independent not only of age, but also of performance status, gender and smoking status. According to the ESMO guidelines (57), the standard of care for tumours bearing an activating EGFR mutation is a first-line treatment with an EGFR 
TKI (erlotinib, gefitinib or afatinib). In patients with asymptomatic BM who have not yet received prior systemic therapy, the use of CNS-penetrant next-generation TKIs (osimertinib, alectinib, ceritinib, brigatinib) may restore control of brain disease, with the potential to delay cranial radiotherapy (57). However, there is some data, albeit of low quality, coming from a meta-analysis (58) and from a multiinstitutional retrospective analysis (59), that upfront cranial radiotherapy may improve intracranial disease control (58) and survival outcomes compared with TKI alone $(58,59)$. The meta-analysis showed the OS benefit at two years with concurrent or sequential upfront WBRT (RR 1.33; 95\% CI: $1.00-1.77, \mathrm{P}=0.05$ ) and sequential upfront SRS (RR 2.08; 95\% CI: $1.67-2.61$ ) compared to TKI alone (58). In a retrospective multi-institutional study SRS $v s$. EGFRTKI and WBRT vs. EGFR-TKI were associated with improved OS: the median OS for the SRS ( $\mathrm{N}=100)$, WBRT $(\mathrm{N}=120)$, and EGFR-TKI $(\mathrm{N}=131)$ cohorts was 46, 30, and 25 months, respectively $(\mathrm{P}<0.001)$ (59). In this study, however, age $>70 v s$. $>50$ years was associated with worse survival in multivariate analysis, $\mathrm{P}=0.028$.

In patients with ALK-rearranged NSCLC and BM, an absence of prior treatment with ALK-targeted TKIs, absence of extracranial disease, and KPS $\geq 90$ were found to be independent predictors of OS, whereas neither age nor smoking history or number of BM, were observed to significantly influence prognosis (60). According to the ESMO guidelines, first-line treatment with a firstgeneration ALK inhibitor, crizotinib is preferred for patients with ALK-rearranged NSCLC (57). However, about 20\% of ALK-positive patients experience brain progression on crizotinib (61). In such patients, treatment with a secondgeneration ALK inhibitor (ceritinib, alectinib or brigatinib), of better intracranial efficacy, is recommended $(57,62)$.

Immunotherapy with checkpoint inhibitorsadministered with CHT or alone-have become a routine part of treatment of NSCLC patients without driver mutations but with $\geq 50 \%$ expression of programmed cell death-ligand 1 (PD-L1) (62). Although to date no studies dedicated to elderly patients have been reported, evidence is accumulating for the use of immune checkpoint inhibitors in the treatment of elderly patients with advanced NSCLCnone of the subgroup analyses of the randomized trials of immune checkpoint inhibitors showed worse efficacy, greater toxicity nor differences in survival benefit between NSCLC patients aged $\leq 65$ and $>65$ years (51). According to the CSCO-ESMO guidelines, immunotherapy should be considered according to standard recommendations in elderly patients (51). However, in NSCLC patients with $\mathrm{BM}$, although immune checkpoint inhibitors have resulted in increased intracranial activity compared to CHT, the evidence is not strong enough to defer local therapy and use them as an upfront therapy.

The combination of radiation therapy and targeted agents represents an opportunity to improve the outcomes of patients with BM, but the safety of this approach is yet to be confirmed within prospective trials, and no data specific for elderly patients is available.

\section{Best supportive care alone}

The benefit of WBRT for patients with poor prognosis continues to be debated. The results of a phase III noninferiority QUARTZ trial, in which NSCLC patients with $\mathrm{BM}$ unsuitable for surgical resection or SRS were randomised to receive either BSC including dexamethasone plus WBRT (20 Gy in 5 daily fractions) or the same BSC without WBRT showed no difference between the treatment arms in terms of symptom relief, steroid use, OS, QoL or quality-adjusted life years, confirming no benefit for WBRT in the RPA Class III subset (63). The OS reported was only 9.2 and 8.5 weeks, for the WBRT and BSC arms, respectively. For older patients ( $\geq 60$ years), WBRT offered no benefit over BSC in terms of either survival or QoL, and in patients older than 70 years WBRT appeared to even worsen OS (HR 0.75, 95\% CI: $0.56-$ 1.00), irrespective of their KPS. According to the ESMO guidelines, radiation therapy is not recommended for RPA Class III patients (with KPS of $\leq 70 \%$ ) based on their dismal prognosis (median survival is generally $<2$ months) (57). Elderly patients with limited life expectancy and/or poor performance status, are unlikely to benefit from WBRT and BSC with corticosteroids alone is reasonable. However, for patients with better prognosis unsuitable for surgical resection or SRS, including the selected elderly patients, there is no conclusive evidence to restrict the use of WBRT, especially for symptomatic BM, which is discussed in more detail below.

\section{QoL and neurocognitive function after RT for BM in the elderly}

QoL is dependent on neurocognitive function after treatment for BM (64). It may be influenced by the mental status before the treatment thus neurocognitive assessment was advocated to be included into initial staging (65). Data on age-related toxicity is scarce, mostly based on 
retrospective trials or subgroup analyses suggesting potentially increased radiation-induced toxicity secondary to comorbidities, impaired functional status and cognitive function at baseline (66).

Some data suggests that subacute brain atrophy associated with neurologic and mental deterioration presented 2-3 months after brain radiotherapy is correlated with older age. Pathologic findings revealed radiation brain atrophy in $72.9 \%$ of patients $>50$ years, in contrast to $39.2 \%$ of patients $<50$ years, for the dose range from 30 Gy (WBRT) to 80 Gy (tumor bed). The average age of patients with post-radiation dementia was 60.5 years comparing to 42.8 years with normal mental status $(\mathrm{P}<0.001)(29)$. Another retrospective study on 111 patients who underwent WBRT for BM revealed that the risk of leukoencephalopathy, meant mainly as an injury of the white matter, causing motor dysfunction, emotional changes, urinary incontinence, seizure and even coma, also increased with age. Age $>65$ years was significantly correlated with higher risk of leukoencephalopathy (odds ratio 3.31; 95\% CI: $1.115-9.5, \mathrm{P}=0.03$ ), with a median time to develop this complication of 5.5 months after WBRT (67).

More robust data on the correlation of neurotoxicity following WBRT with age comes from the phase II RTOG0212 study on the dose in PCI for SCLC. Primary analysis of this trial revealed that increasing age was the most significant predictor of chronic neurotoxicity after PCI $(\mathrm{P}=0.0005)$ (65). It was confirmed by the analysis of QoL and neurological data from the same trial. Physical functioning, motor dysfunction, Lent Soma memory test results and MRI and/or CT abnormalities worsened with age. Patients $>60$ years were more likely to experience neurotoxicity 12 months after PCI: $83 \%$ vs. $56 \%, \mathrm{P}=0.009$ (24). On the other hand, an analysis of the impact of PCI on neurocognitive function and QoL in the RTOG-0214 trial on PCI $v s$. observation in NSCLC patients revealed that no clear differences at 1 year emerged in neurocognitive function or QoL between patients $\leq 60$ or older than 60 years on either arm (all adjusted $\mathrm{P}<0.05)(25)$.

We may expect that neurotoxicity will be reduced in the elderly by using ablative techniques with omission of WBRT. However, age-specific toxicity is under-reported in the SRS studies. Gregucci et al. (68) reported no grade $>2$ neurologic adverse events in a group of 40 patients older than 65 years treated with SRS and stereotactic fractionated radiotherapy for less than ten BM. SRS for BM was also studied in patients older than 80 years versus $65-75$ years old (4). The incidences of neurological death and deterioration were insignificantly lower in the older group, and the incidence of SRS related complications reported using RTOG toxicity scale was similar in both groups (HR 0.616, 95\% CI: 0.152-2.495, $\mathrm{P}=0.49$ ) (4). Chen et al. (8) revealed that among patients 70-79 years $v s$. older than 80 years, treated with RT for BM, acute RTOG grade 1-4 toxicity occurred in $89 \%$ of WBRT-treated patients and in $68 \%$ of SRS-treated patients, and that the main impact on toxicity in this group of patients was caused by the treatment modality and not age.

All these data suggest that some neurological side effects can be diminished with a smaller volume irradiated.

\section{NSCLC}

\section{BM suitable for either surgery or SRS}

Outside recognized indications for surgery such as establishing diagnosis or relieving mass effect, little evidence is available to guide the therapeutic choice of SRS $v s$. surgical resection in the treatment of patients with limited BM (69). No prospective randomised trials comparing SRS $v s$. surgery was performed, and most of the retrospective studies reported similar outcomes in brain control or survival in solitary resectable brain metastases $(70,71)$. In a secondary analysis of patients treated with SRS $v s$. surgical resection within the EORTC 22952-26001 phase III trial, Churilla et al. assessed local control of treated BM after adjustment for tumor size, metastasis site and number, neurologic status, and the presence of extracranial disease, revealing that although early local control favored SRS, the advantage diminished in magnitude over time with more late recurrences observed in the SRS group (69). However, in case of the elderly, with limited survival prognosis and possibly higher risk of postoperative morbidity, SRS may be the optimal choice when technically feasible and when there is no indication for surgical decompression or need for histologic diagnosis. High rates of tumor bed recurrence after surgical resection of BM without adjuvant brain treatment $(20,72)$ in view of the recognized neurotoxicity of WBRT have led to an interest in post-operative SRS to resection cavities. However, taking into account the lack of unequivocal evidence of survival benefit from adjuvant treatment and conflicting results of two randomized trials performed to date comparing post-operative SRS $v s$. WBRT $(73,74)$ none of which provided data on the influence of age on the outcomes, use of the post-operative treatment in the elderly is questionable. Close MRI brain imaging follow-up seems to be a reasonable option. 


\section{BM unsuitable for either surgery or SRS: for whom-if any-WBRT?}

The role of WBRT in unselected NSCLC patients not suitable for SRS or surgery has been questioned by the results of the QUARTZ trial (63), which suggest that WBRT can be omitted and patients treated with BSC alone, without an important reduction in either OS or QoL. Improved survival with WBRT was shown for younger patients, particularly those aged younger than 60 years and there were also non-significant associations suggesting a potential survival benefit with WBRT for patients with good performance status and a controlled primary NSCLC (63). The results of this trial have been questioned, mostly due to unexpectedly poor outcomes in the whole cohort and a very small percentage of patients with better prognosis (RPA Class I in $8 \%$ and $3 \%$ of the WBRT and BSC arm, respectively) $(75,76)$. However, these results have been supported by a large retrospective analysis of all patients with BM from NSCLC $(\mathrm{N}=4,363)$ or breast cancer $(\mathrm{N}=1,962)$ treated with WBRT in 15 out of 21 Dutch radiotherapy centers between 2000 and 2014 (77). The survival of patients after WBRT for BM from NSCLC was poor, especially in the elderly. In the whole cohort, patients older than 70 years had significantly worse survival, HR 1.34, 95\% CI: $1.23-1.47, \mathrm{P}<0.001$; for NSCLC patients median survival was 2.7 months (95\% CI: 2.6-2.8), whereas in patients older than 70 years $(\mathrm{N}=1,118)$ median OS was 2.1 months (95\% CI: 2.2-2.3). The median age in the study group (62 years) was similar to that in the QUARTZ trial (66 years), and the impact of age on survival seemed larger in NSCLC compared to breast cancer (77). These results, suggesting very poor prognosis in patients with BM from NSCLC treated with WBRT, are in line not only with the results of the QUARTZ trial, but also with the cohort trials $(78,79)$, in contrast to the prospective clinical trials on BM treated with WBRT in NSCLC where survival tends to be higher, with a median of 5.2-7.2 months and even higher when combined with systemic therapy (80). Based on their results being in line with the results presented in the QUARTZ trial the authors advocated a much more restrictive use of WBRT. However, they speculated that a possible explanation for the poor survival in this cohort could be that with the increase of SRS, patients currently treated with WBRT are more likely to be at the end stage of the disease than in the past, and only a few will receive systemic treatment (77). Taking into account a wide heterogeneity in prognosis for patients with
BM from NSCLC [e.g., survival in patients with EGFR- or ALK- mutated NSCLC has been increased by at least three times over the past 15 years (75)], the question still remains whether the use of WBRT is definitely ruled out in NSCLC patients. Younger patients, with controlled extracranial disease, i.e., RPA Class I patients and a more favorable subset of patients within RPA Class II, do appear to benefit from WBRT (76). This second subset, i.e., RPA Class II patients, consists also of selected elderly patients, for whom the evidence to restrict the use of WBRT are inconclusive, although the optimal treatment strategy remains to be determined, ideally in a prospective randomized trial.

\section{SCLC}

\section{Treatment of BM with respect to feasibility of $\mathrm{CHT}$}

CHT is a mainstay of treatment of SCLC, however the specificity of the elderly subgroup with variable performance status and comorbidities often limits CHT options. Elderly patients fit enough for CHT with asymptomatic BM should receive the upfront $\mathrm{CHT}$. As in the aforementioned analysis of 1,615 SCLC patients $\geq 75$ years old form the USA national cancer database (NCDB) no OS benefit from WBRT in patients that received CHT (49) was suggested, CHT alone may be considered as an option for this subgroup. However, there is no evidence from randomized studies on the role of $\mathrm{CHT}$ alone in the exclusively geriatric population. Since CHT without WBRT leads to a significantly shorter time to progression in the brain $(50,81)$, a well selected elderly patient population with better prognosis (excellent performance status, limited asymptomatic BM and control of extracranial disease on CHT) may possibly benefit from some form of radiation. As such patients have a chance to live long enough to experience the possible cognitive effects of WBRT, SRS emerges as the optimal treatment option. Another NCDB-based study on 5,952 investigating SCLC patients treated with upfront WBRT $(\mathrm{N}=5,752)$ vs. upfront SRS alone $(\mathrm{N}=200)$ showed that upfront SRS was associated with superior OS (median 10.8 vs. 7.1 months, $\mathrm{P}<0.001$ ) which may suggest that SRS-alone may be appropriate for some patients with SCLC (82). However, age $>65$ years was associated with worse OS in this study (HR 1.419, 95\% CI: $1.345-1.496, \mathrm{P}<0.001)$. The safety and benefit of the SRS with omission of WBRT in BM from SCLC remains to be confirmed in a prospective trial.

For patients with symptomatic BM who are fit enough 
for CHT, standard practice is to use upfront WBRT, to palliate the symptoms and improve patient's condition before the start of CHT.

Elderly patients not fit enough for CHT have particularly poor prognosis. Median OS for such patients was 1.9 months with WBRT vs. 1.2 months without WBRT $(\mathrm{P}<0.0001)$ in the NCDB-based analysis of 1,615 elderly patients with BM from SCLC (49). Unfortunately, no data on the performance status of these patients - being, after all, the strongest predictor of survival—was reported. With such a short survival, the benefit of WBRT must be considered doubtful. However, this modest, albeit statistically significant, 15 day survival benefit with the use of WBRT is in line with the results of another study, on 113 patients with poor prognosis with different primary tumors managed with BSC vs. WBRT, which revealed that improved survival after WBRT was limited to patients with primary SCLC (median survival of 0.6 vs. 5.3 months for BSC vs. WBRT, respectively, $\mathrm{P}=0.001$ ) (83). The true benefit of WBRT, residing in its ability to palliate symptoms, which may be more efficient for SCLC than for other histologies, due to the radio responsive nature of SCLC (49). For this reason, extrapolating the results of the QUARTZ trial on the SCLC patients may be misleading $(49,83)$. However, no data on the impact of WBRT on QoL in elderly patients with BM from SCLC was reported to date. Further studies are necessary to confirm whether management of SCLC patients should include a less restricted use of WBRT.

A specific subgroup of SCLC patients not feasible for CHT is RTOG RPA Class III, that is, patients with poor performance status (KPS <70). There is no data on the role of WBRT $v s$. BSC specific for the geriatric population of such patients. For the unselected RTOG RPA Class III patients, one prospective trial which also included patients with SCLC histology aimed to determine whether WBRT had any benefit on symptom palliation (84). Ninety-one patients received WBRT and were asked to complete a questionnaire about their symptoms before and 1 month after WBRT. In patients who completed both questionnaires, the intensity of symptoms significantly increased after WBRT. In addition to the results of the QUARTZ study (63), this data also challenges the value of WBRT for SCLC patients with poor performance status.

\section{Treatment of BM with respect to prophylactic cranial irradiation (PCI)}

In patients with symptomatic BM occurring during firstline CHT without prior use of PCI, WBRT is usually given, then CHT may be continued or not depending on the systemic CHT response and performance status of the patient (48). In such a scenario, elderly patients receiving CHT should also be managed this way.

In elderly patients with no prior PCI and metachronous $\mathrm{BM}$ occurring after completion of CHT, second line CHT is very rarely feasible. WBRT remains the standard therapeutic strategy for these patients, apart from RPA Class III patients, for whom BSC with steroids alone is a preferred option.

In elderly patients who have developed metachronous $\mathrm{BM}$ after prior PCI, the most appropriate salvage treatment option for these with better prognosis (i.e., life expectancy of more than 3 months) is SRS, when technically feasible. In a series of 13 patients re-irradiated with SRS after a median of 14 months from the prior PCI, median survival was 5 months with no radionecrosis recorded (85). Age of the patients in this group was not reported, so extrapolating these results to the elderly population may be misleading. In other reports on reirradiation with SRS, median OS after SRS was 3 to 9 months, which must be interpreted taking into account the selection bias, seriously impacting such retrospective analyses (86-92). The risk of radionecrosis reported in these trials was from $4.3 \%$ (2 of 47 patients) (87) to $12.5 \%$ (5 of 40) (86), but no data specific for the elderly was provided. One of these studies reported that age $\geq 65$ years was not predictive for functional preservation (92). However, not only distant brain control but also local control after SRS for BM from SCLC was reported to be lower than for BM from other solid tumors with oneyear local control rate of $<70 \%$ for SCLC $(86,87)$ in contrast to $70-90 \%$ reported in prospective trials for other histologies $(18,20)$. Nevertheless, most SCLC patients recur as multifocal BM not practically addressed with focal therapies (92). There are very few reports about whole brain reirradiation after PCI, and no data specific to the elderly. The risk that late toxicity would manifest in patients with such a short life expectancy should be considered unlikely, and the short term effects of reirradiation at moderate doses may be beneficial, especially in symptomatic patients, taking into account the radiosensitivity of SCLC. The largest series of 66 patients treated with WBRT after PCI showed symptom improvement in $40 \%$ of patients and no serious, grade $>2$ toxicity ( 85 ). Older age ( $>60$ years) was not associated with worse survival in this.

\section{Conclusions}

Treatment of the elderly patients with BM from lung cancer 
is not based on the evidence from randomized prospective trials. Age is a well recognized poor prognostic factor for survival in patients with BM from lung cancer, which is reflected in prognostic scales, but its impact on the patients' prognosis reflected by its value in gradually updated grading indices is decreasing.

For elderly NSCLC patients with asymptomatic BM, first-line treatment with CHT and deferred WBRT should be considered. In patients with driver mutations with clinically asymptomatic BM, TT-having far greater intracranial efficacy than conventional CHT-may restore control of brain disease and delay cranial radiotherapy, which is of importance in the elderly, being more susceptible to radiation-induced neurotoxicity. SRS is a reasonable approach for older patients with a limited number of $\mathrm{BM}$ from NSCLC, with both survival benefit and toxicity profile similar to those observed in young adults. Selected elderly NSCLC patients with BM unsuitable for surgical resection or SRS and more favorable prognosis (i.e., RPA Class II patients), especially symptomatic, may benefit from WBRT.

For the elderly SCLC patients with newly diagnosed asymptomatic BM CHT alone may be a reasonable strategy, adding radiotherapy to CHT results in significantly prolonged time to progression within the brain, but with no impact on OS. SRS is an attractive treatment option for $\mathrm{BM}$ occurring after prior PCI and should be used whenever technically feasible; however, WBRT at moderate doses is also feasible. WBRT is the treatment of first choice in patients who are unsuitable for SRS or symptomatic patients.

For RPA Class III patients, regardless of histology, BSC with steroids alone is a preferred option.

The impact of different radiation techniques and regimens incorporating RT, CHT and/or TT on survival, neurocognitive outcome and QoL in this specific subgroup still need to be evaluated, preferably in a prospective trial.

\section{Acknowledgments}

Funding: None.

\section{Footnote}

Provenance and Peer Review: This article was commissioned by the editorial office, Fournal of Thoracic Disease for the series "Radiotherapy for Brain Metastases from Lung Cancer". The article has undergone external peer review.
Conflicts of Interest: All authors have completed the ICMJE uniform disclosure form (available at http:// dx.doi.org/10.21037/jtd-2019-rbmlc-05). The series "Radiotherapy for Brain Metastases from Lung Cancer" was commissioned by the editorial office without any funding or sponsorship. LK served as the unpaid Guest Editor of the series and serves as an unpaid editorial board member of Fournal of Thoracic Disease from Aug 2018 to Jul 2020. The authors have no other conflicts of interest to declare.

Ethical Statement: The authors are accountable for all aspects of the work in ensuring that questions related to the accuracy or integrity of any part of the work are appropriately investigated and resolved.

Open Access Statement: This is an Open Access article distributed in accordance with the Creative Commons Attribution-NonCommercial-NoDerivs 4.0 International License (CC BY-NC-ND 4.0), which permits the noncommercial replication and distribution of the article with the strict proviso that no changes or edits are made and the original work is properly cited (including links to both the formal publication through the relevant DOI and the license). See: https://creativecommons.org/licenses/by-nc-nd/4.0/.

\section{References}

1. Fox BD, Cheung VJ, Patel AJ, et al. Epidemiology of metastatic brain tumors. Neurosurg Clin N Am 2011;22:1-6.

2. Yancik R, Ries LA. Cancer in older persons: an international issue in an aging world. Semin Oncol 2004;31:128-36.

3. Ascha MS, Ostrom QT, Wright J, et al. Lifetime Occurrence of Brain Metastases Arising from Lung, Breast, and Skin Cancers in the Elderly: A SEER-Medicare Study. Cancer Epidemiol Biomarkers Prev 2019;28:917-25.

4. Watanabe S, Yamamoto M, Sato Y et al. Stereotactic radiosurgery for brain metastases: a case-matched study comparing treatment results for patients 80 years of age or older versus patients 65-79 years of age. J Neurosurg 2014;121:1148-57.

5. Gaspar L, Scott C, Rotman M, et al. Recursive partitioning analysis (RPA) of prognostic factors in three Radiation Therapy Oncology Group (RTOG) brain metastases trials. Int J Radiat Oncol Biol Phys 1997;37:745-51.

6. Sperduto PW, Yang TJ, Beal K, et al. Estimating Survival 
in Patients With Lung Cancer and Brain Metastases: An Update of the Graded Prognostic Assessment for Lung Cancer Using Molecular Markers (Lung-molGPA). JAMA Oncol 2017;3:827-31.

7. Yomo S, Hayashi M. Is upfront stereotactic radiosurgery a rational treatment option for very elderly patients with brain metastases? A retrospective analysis of 106 consecutive patients age 80 years and older. BMC Cancer 2016;16:948.

8. Chen L, Shen C, Redmond KJ, et al: Use of stereotactic radiosurgery in elderly and very elderly patients with brain metastases to limit toxicity associated with whole brain radiation therapy. Int J Radiat Oncol Biol Phys 2017;98:939-47.

9. Sperduto PW, Berkey B, Gaspar LE, et al. A new prognostic index and comparison to three other indices for patients with brain metastases: an analysis of 1,960 patients in the RTOG database. Int J Radiat Oncol Biol Phys 2008;70:510-4.

10. Nadpara PA, Madhavan SS, Tworek C, et al. Guidelineconcordant lung cancer care and associated health outcomes among elderly patients in the United States. J Geriatr Oncol 2015;6:101-10.

11. Bakogeorgos M, Mountzios G, Bournakis E, et al. Do elderly patients with non-small cell lung cancer get the best out of recent advances in first-line treatment? A comparative study in two tertiary cancer centers in Greece. J Geriatr Oncol 2015;6:111-8.

12. Erb CT, Su KW, Soulos PR, et al. Surveillance Practice Patterns after Curative Intent Therapy for Stage I NonSmall-Cell Lung Cancer in the Medicare Population. Lung Cancer 2016;99:200-7.

13. Weltman E, Salvajoli JV, Brandt RA, et al. Radiosurgery for brain metastases: A score index for predicting prognosis. Int J Radiat Oncol Biol Phys 2000;46:1155-61.

14. Lorenzoni J, Devriendt D, Massager N, et al. Radiosurgery for treatment of brain metastases: Estimation of patient eligibility using three stratification systems. Int J Radiat Oncol Biol Phys 2004;60:218-24.

15. Sperduto PW, Kased N, Roberge D, et al. Summary report on the graded prognostic assessment: an accurate and facile diagnosis-specific tool to estimate survival for patients with brain metastases. J Clin Oncol 2012;30:419-25.

16. Bernhardt D, König L, Aufderstrasse S, et al. Generation of a New Disease-specific Prognostic Score for Patients With Brain Metastases From Small-cell Lung Cancer Treated With Whole Brain Radiotherapy (BMS-Score) and Validation of Two Other Indices. Clin Lung Cancer
2018;19:340-5.

17. Yamamoto M, Serizawa T, Higuchi Y, et al. Prognostic grading system specifically for elderly patients with brain metastases after stereotactic radiosurgery: a 2-institution study. J Neurosurg 2018;129:95-102.

18. Aoyama H, Shirato H, Tago M, et al. Stereotactic radiosurgery plus whole-brain radiation therapy vs stereotactic radiosurgery alone for treatment of brain metastases: a randomized controlled trial. JAMA 2006;295:2483-91.

19. Chang EL, Wefel JS, Hess KR, et al. Neurocognition in patients with brain metastases treated with radiosurgery or radiosurgery plus whole-brain irradiation: a randomised controlled trial. Lancet Oncol 2009;10:1037-44.

20. Kocher M, Soffietti R, Abacioglu U, et al. Adjuvant wholebrain radiotherapy versus observation after radiosurgery or surgical resection of one to three cerebral metastases: results of the EORTC 22952-26001 study. J Clin Oncol 2011;29:134-41.

21. Andrews DW, Scott CB, Sperduto PW, et al. Whole brain radiation therapy with or without stereotactic radiosurgery boost for patients with one to three brain metastases: phase III results of the RTOG 9508 randomised trial. Lancet 2004;363:1665-72.

22. Sperduto PW, Shanley R, Luo X, et al. Secondary analysis of RTOG 9508, a phase 3 randomized trial of wholebrain radiation therapy versus $W B R T$ plus stereotactic radiosurgery in patients with 1-3 brain metastases; poststratified by the graded prognostic assessment (GPA). Int J Radiat Oncol Biol Phys 2014;90:526-31.

23. Crossen JR, Garwood D, Glatstein E, et al.

Neurobehavioral sequelae of cranial irradiation in adults: a review of radiation-induced encephalopathy. J Clin Oncol 1994;12:627-42.

24. Le Péchoux C, Laplanche A, Faivre-Finn C, et al. Prophylactic Cranial Irradiation (PCI) Collaborative Group. Clinical neurological outcome and quality of life among patients with limited small cell cancer treated with two different doses of prophylactic cranial irradiation in the intergroup phase III trial (PCI99-01, EORTC 22003-08004,RTOG 0212 and IFCT 99-01). Ann Oncol 2011;22:1154-63.

25. Sun A, Bae K, Gore EM, et al. Phase III trial of prophylactic cranial irradiation compared with observation in patients with locally advanced non-small-cell lung cancer: neurocognitive and quality-of life analysis. J Clin Oncol 2011;29:279-86.

26. Brown PD, Jaeckle K, Ballman KV, et al. Effect of 
radiosurgery alone vs RadiosurgeryWith whole brain radiation therapy on cognitive function in patients with 1 to 3 brain metastases: a randomized clinical trial. JAMA 2016;316:401-9.

27. Wong J, Hird A, Zhang L, et al. Symptoms and quality of life in cancer patients with brain metastases following palliative radiotherapy. Int J Radiat Oncol Biol Phys 2009;75:1125-31.

28. Soffietti R, Kocher M, Abacioglu UM, et al. A European organisation for research and treatment of cancer phase III trial of adjuvant whole-brain radiotherapy versus observation in patients with one to three brain metastases from solid tumors after surgical resection or radiosurgery: quality-of-life results. J Clin Oncol 2013;31:65-72.

29. Asai A, Matsutani M, Kohno T, et al. Subacute brain atrophy after radiation therapy for malignant brain tumor. Cancer 1989;63:1962-74.

30. Noel G, Bollet MA, Noel S, et al. Linac stereotactic radiosurgery: an effective and safe treatment for elderly patients with brain metastases. Int J Radiat Oncol Biol Phys 2005;63:1555-61.

31. Minniti G, Esposito V, Clarke E, et al: Stereotactic radiosurgery in elderly patients with brain metastases. J Neurooncol 2013;111:319-25.

32. Park JY, Moon KS, Lee KH, et al. Gamma knife radiosurgery for elderly patients with brain metastases: evaluation of scoring systems that predict survival. BMC Cancer 2015;15:54.

33. Yamamoto M, Serizawa T, Shuto T et al. Stereotactic radiosurgery for patients with multiple brain metastases (JLGK0901): a multi-institutional prospective observational study. Lancet Oncol 2014;15:387-95.

34. Higuchi $Y$, Yamamoto $M$, Serizawa T, et al. Stereotactic radiosurgery in elderly patients with brain metastases: comparison with non-elderly patients using database of a multi-institutional prospective observational study (JLGK0901-Elderly). J Neurooncol 2019;144:393-402.

35. Minniti G, Clarke E, Lanzetta G, et al. Stereotactic radiosurgery for brain metastases: analysis of outcome and risk of brain radionecrosis. Radiat Oncol 2011;6:48.

36. Lawrence YR, Li XA, el Naqa I, et al. Radiation dosevolume effects in the brain. Int J Radiat Oncol Biol Phys 2010;76:S20-7.

37. Zhuang H, Zheng Y, Wang J, et al. Analysis of risk and predictors of brain radiation necrosis after radiosurgery. Oncotarget 2016;7:7773-9.

38. Kim JM, Miller JA, Kotecha R, et al. The risk of radiation necrosis following stereotactic radiosurgery with concurrent systemic therapies. J Neurooncol 2017;133:357-68.

39. Miller JA, Bennett EE, Xiao R, et al. Association Between Radiation Necrosis and Tumor Biology After Stereotactic Radiosurgery for Brain Metastasis. Int J Radiat Oncol Biol Phys 2016;96:1060-9.

40. Patchell RA, Tibbs PA, Walsh JW, et al. A randomized trial of surgery in the treatment of single metastases to the brain.N Engl J Med1990;322:494-500.

41. Vecht CJ, Haaxma-Reiche H, Noordijk EM, et al. Treatment of single brain metastasis: radiotherapy alone or combined with neurosurgery? Ann Neurol1993;33:583-90.

42. Noordijk EM, Vecht CJ, Haaxma-Reiche H, et al. The choice of treatment of single brain metastasis should be based on extracranial tumor activity and age. Int J Radiat Oncol Biol Phys 1994;29:711-7.

43. Grossman R, Mukherjee D, Chang D, et al. Predictors of inpatient death and complications among postoperative elderly patients with metastatic brain tumors. Ann Surg Oncol 2011;18:521-8.

44. Zimmermann S, Dziadziuszko R, Peters S. Indications and limitations of chemotherapy and targeted agents in nonsmall cell lung cancer brain metastases. Cancer Treat Rev 2014;40:716-22.

45. Reck M, Popat S, Reinmuth N, et al. Metastatic nonsmall-cell lung cancer (NSCLC): ESMO Clinical Practice Guidelines for diagnosis, treatment and follow-up. Ann Oncol 2014;25 Suppl 3:iii27-39.

46. Grossi F, Scolaro T, Tixi L, et al. The role of systemic chemotherapy in the treatment of brain metastases from small-cell lung cancer. Crit Rev Oncol Hematol 2001;37:61-7.

47. Seute T, Leffers P, Wilmink JT, et al. Response of symptomatic brain metastases from small-cell lung cancer to systemic first-line chemotherapy. J Clin Oncol 2006;24:2079-83.

48. Kepka L. Radiotherapy of brain metastases from smallcell lung cancer: standards and controversies. J Cancer Metastasis Treat 2019;5:53.

49. Renz P, Hasan S, Wegner RE. Survival outcomes after whole brain radiotherapy for brain metastases in older adults with newly diagnosed metastatic small cell carcinoma: A national cancer database (NCDB) analysis. J Geriatr Oncol 2019;10:560-6.

50. Postmus PE, Haaxma-Reiche H, Smit EF, et al. Treatment of brain metastases of small-cell lung cancer: comparing teniposide and teniposide with whole-brain radiotherapy-a phase III study of the European Organization for 
the Research and Treatment of Cancer Lung Cancer Cooperative Group. J Clin Oncol 2000;18:3400-8.

51. Wu YL, Planchard D, Lu S, et al. Pan-Asian adapted Clinical Practice Guidelines for the management of patients with metastatic non-small-cell lung cancer: a CSCO-ESMO initiative endorsed by JSMO, KSMO, MOS, SSO and TOS. Ann Oncol 2019;30:171-210.

52. Han JY, Park K, Kim SW et al. First-SIGNAL: first-line single-agent iressa versus gemcitabine and cisplatin trial in never-smokers with adenocarcinoma of the lung. J Clin Oncol 2012;30:1122-8.

53. Maemondo M, Inoue A, Kobayashi K et al. Gefitinib or chemotherapy for non-small-cell lung cancer with mutated EGFR. N Engl J Med 2010;362:2380-8.

54. Mok TS, Wu YL, Thongprasert S et al. Gefitinib or carboplatin-paclitaxel in pulmonary adenocarcinoma. N Engl J Med 2009;361:947-57.

55. Rosell R, Carcereny E, Gervais R et al. Erlotinib versus standard chemotherapy as first-line treatment for European patients with advanced EGFR mutation-positive non-small-cell lung cancer (EURTAC): a multicentre, open-label, randomised phase 3 trial. Lancet Oncol 2012;13:239-46.

56. Sequist LV, Yang JC, Yamamoto $\mathrm{N}$ et al. Phase III study of afatinib or cisplatin plus pemetrexed in patients with metastatic lung adenocarcinoma with EGFR mutations. J Clin Oncol 2013;31:3327-34.

57. Novello S, Barlesi F, Califano R, et al. Metastatic nonsmall-cell lung cancer: ESMO Clinical Practice Guidelines for diagnosis, treatment and follow-up. Ann Oncol 2016;27:v1-v27.

58. Soon YY, Leong CN, Koh WY, et al. EGFR tyrosine kinase inhibitors versus cranial radiation therapy for EGFR mutant non-small cell lung cancer with brain metastases: a systematic review and meta-analysis. Radiother Oncol 2015;114:167-72.

59. Magnuson WJ, Lester-Coll NH, Wu AJ, et al. Management of brain metastases in tyrosine kinase inhibitor-naive epidermal growth factor receptor-mutant non-small-cell lung cancer: A Retrospective MultiInstitutional Analysis. J Clin Oncol 2017;35:1070-7.

60. Johung KL, Yeh N, Desai NB, et al. Extended Survival and Prognostic Factors for Patients With ALK-Rearranged Non-Small-Cell Lung Cancer and Brain Metastasis. J Clin Oncol 2016;34:123-9.

61. Costa DB, Shaw AT, Ou SH, et al. Clinical Experience With Crizotinib in Patients With Advanced ALKRearranged Non-Small-Cell Lung Cancer and Brain
Metastases. J Clin Oncol 2015;33:1881-8.

62. NCCN Clinical Practice Guidelines in Oncology. NonSmall Cell Lung Cancer, Version 324 2.2019. https://www. nccn.org, accessesed on August 31st, 2019.

63. Mulvenna P, Nankivell M, Barton R et al. Dexamethasone and supportive care with or without whole brain radiotherapy in treating patients with non-small cell lung cancer with brain metastases unsuitable for resection or stereotactic radiotherapy (QUARTZ): results from a phase 3 , non-inferiority, randomised trial. Lancet 2016;388:2004-14.

64. Li J, Bentzen SM, Li J, et al. Relationship between neurocognitive function and quality of life after wholebrain radiotherapy in patients with brain metastasis. Int J Radiat Oncol Biol Phys 2008;71:64-70.

65. Wolfson AH, Bae K, Komaki R, et al. Primary analysis of a phase II randomized trial Radiation Therapy Oncology Group (RTOG) 0212: impact of different total doses and schedules of prophylactic cranial irradiation on chronic neurotoxicity and quality of life for patients with limiteddisease small-cell lung cancer. Int J Radiat Oncol Biol Phys 2011;81:77-84.

66. Minniti G, Filippi AR, Osti MF, et al. Radiation therapy for older patients with brain tumors. Radiat Oncol 2017;12:101.

67. Ebi J, Sato H, Nakajima M, et al. Incidence of leukoencephalopathy after whole-brain radiation therapy for brain metastases. Int J Radiat Oncol Biol Phys 2013;85:1212-7.

68. Gregucci F, Fiorentino A, Corradini S, et al. Linac-based radiosurgery or fractionated stereotactic radiotherapy with flattening filter-free volumetric modulated arc therapy in elderly patients : A mono-institutional experience on 110 brain metastases. Strahlenther Onkol 2019;195:218-25.

69. Churilla TM, Chowdhury IH, Handorf E, et al. Comparison of Local Control of Brain Metastases With Stereotactic Radiosurgery vs Surgical Resection: A Secondary Analysis of a Randomized Clinical Trial. JAMA Oncol 2019;5:243-7.

70. Auchter RM, Lamond JP, Alexander E, et al. A multiinstitutional outcome and prognostic factor analysis of radiosurgery for resectable single brain metastasis. Int J Radiat Oncol Biol Phys 1996;35:27-35.

71. Muacevic A, Kreth FW, Horstmann GA, et al. Surgery and radiotherapy compared with gamma knife radiosurgery in the treatment of solitary cerebral metastases of small diameter. J Neurosurg 1999;91:35-43.

72. Mahajan A, Ahmed S, McAleer MF, et al. Post-operative 
stereotactic radiosurgery versus observation for completely resected brain metastases: a single-centre, randomised, controlled, phase 3 trial. Lancet Oncol 2017;18:1040-8.

73. Kępka L, Tyc-Szczepaniak D, Bujko K, et al. Stereotactic radiotherapy of the tumor bed compared to whole brain radiotherapy after surgery of single brain metastasis: Results from a randomized trial. Radiother Oncol 2016;121:217-24.

74. Brown PD, Ballman KV, Cerhan JH, et al. Postoperative stereotactic radiosurgery compared with whole brain radiotherapy for resected metastatic brain disease (NCCTG N107C/CEC.3): a multicentre, randomised, controlled, phase 3 trial. Lancet Oncol 2017;18:1049-60.

75. Pechoux CL, Dhermain F, Besse B. Whole brain radiotherapy in patients with NSCLC and brain metastases. Lancet 2016;388:1960-2.

76. Bruynzeel AM, Lagerwaard FJ. Whole brain radiotherapy for brain metastases from non-small cell lung cancer: the end of an era? J Thorac Dis 2016;8:E1525-7.

77. Jeene PM, de Vries KC, van Nes JGH, et al. Survival after whole brain radiotherapy for brain metastases from lung cancer and breast cancer is poor in 6325 Dutch patients treated between 2000 and 2014. Acta Oncol 2018;57:637-43.

78. Wong E, Tsao M, Zhang L, et al. Survival of patients with multiple brain metastases treated with whole-brain radiotherapy. CNS Oncol 2015;4:213-24.

79. Windsor AA, Koh E-S, Allen S, et al. Poor outcomes after whole brain radiotherapy in patients with brain metastases: results from an international multicentre cohort study. Clin Oncol (R Coll Radiol) 2013;25:674-80.

80. Peters S, Bexelius C, Munk V, et al. The impact of brain metastasis on quality of life, resource utilization and survival in patients with non-small-cell lung cancer. Cancer Treat Rev 2016;45:139-62.

81. Liu Y, Liu X-H, Wang Y, et al. A study on different therapies and prognosis-related factors for 101 patients with SCLC and brain metastases. Cancer Biol Ther 2017;18:670-5.

82. Robin TP, Jones BL, Amini A, et al. Radiosurgery alone is associated with favorable outcomes for brain metastases from small-cell lung cancer. Lung Cancer 2018;120:88-90.

83. Nieder C, Norum J, Dalhaug A, et al. Radiotherapy versus best supportive care in patients with brain metastases and adverse prognostic factors. Clin Exp Metastasis 2013;30:723-9.

84. Komosinska K, Kepka L, Niwinska A, et al. Prospective evaluation of the palliative effect of whole-brain radiotherapy in patients with brain metastases and poor performance status. Acta Oncol 2010;49:382-8.

85. Bernhardt D, Bozorgmehr F, Adeberg S, et al. Outcome in patients with small cell lung cancer re-irradiated for brain metastases after prior prophylactic cranial irradiation. Lung Cancer 2016;101:76-81.

86. Rava P, Sioshansi S, DiPetrillo T, et al. Local recurrence and survival following stereotactic radiosurgery for brain metastases from small cell lung cancer. Pract Radiat Oncol 2015;5:e37-44.

87. Harris S, Chan MD, Lovato JF, et al. Gamma knife stereotactic radiosurgery as salvage therapy after failure of whole-brain radiotherapy in patients with small cell lung cancer. Int J Radiat Oncol Biol Phys 2012;83:e53-9.

88. Yomo S, Hayashi M. Is stereotactic radiosurgery a rational treatment option for brain metastases from small cell lung cancer? A retrospective analysis of 70 consecutive patients. BMC Cancer 2015;15:95.

89. Olson AC, Wegner RE, Rwigema JCM, et al. Clinical outcomes of reirradiation of brain metastases from small cell lung cancer with cyberknife stereotactic radiosurgery. J Cancer Res Ther 2012;8:411-6.

90. Wegner RE, Olson AC, Kondziolka D, et al. Stereotactic radiosurgery for patients with brain metastases from small cell lung cancer. Int J Radiat Oncol Biol Phys 2011;81:e21-7.

91. Nakazaki K, Higuchi Y, Nagano O et al. Efficacy and limitations of salvage gamma knife radiosurgery for brain metastases of small-cell lung cancer after whole-brain radiotherapy. Acta Neurochir 2013;155:107-13.

92. Mazure B, Guest N, Letcher A, et al. Should stereotactic radiosurgery be considered for salvage of intracranial recurrence in small cell lung cancer? J Thorac Oncol 2018;13:S436.
Cite this article as: Socha J, Rychter A, Kepka L. Management of brain metastases in elderly patients with lung cancer. J Thorac Dis 2021;13(5):3295-3307. doi: 10.21037/jtd-2019rbmlc-05 